\title{
Publisher's Note: Pure density functional for strong correlation and the thermodynamic limit from machine learning [Phys. Rev. B 94, 245129 (2016)]
}

\author{
Li Li (李力), Thomas E. Baker®, Steven R. White, and Kieron Burke \\ (Q) (Received 19 March 2021; published 24 March 2021)
}

DOI: 10.1103/PhysRevB.103.119903

This paper was published online on 21 December 2016 with a typographical error in the Acknowledgments. In the Acknowledgments, the first sentence should read as "This work was supported by the U.S. Department of Energy, Office of Science, Basic Energy Sciences under Award No. DE-SC0008696." The Acknowledgments have been corrected as of 17 March 2021. The Acknowledgments are incorrect in the printed version of the journal. 